\title{
Apoptotic factors in physiological and pathological processes of teeth and periodontal tissues - literature review
}

\author{
Urszula Orzedala-Koszel ${ }^{1 \star}$, Mansur Rahnama $^{1}$, Michal Lobacz ${ }^{1}$, Hanna Koszel ${ }^{2}$
}

\author{
${ }^{1}$ Department of Oral Surgery, Medical University of Lublin, 7 Karmelicka, 20-081 Lublin, Poland \\ ${ }^{2}$ Student Research Group, Dept. of Oral Surgery, Medical University of Lublin
}

\begin{tabular}{|c|c|}
\hline ARTICLE INFO & ABSTRACT \\
\hline Received 03 November 2014 & \multirow{3}{*}{$\begin{array}{l}\text { Apoptosis is a physiological process that occurs in the human body throughout the entire } \\
\text { life span. This process can be seen in the tissues of the stomatognathic system. A disorder } \\
\text { in such programmed cell death processes leads to the development of pathological lesions. } \\
\text { Among these are inflammation, osteolytic lesions and neoplastic hyperplasia. We put } \\
\text { forward that future studies should concentrate on how to use the knowledge of apoptotic } \\
\text { processes and their inhibitors in therapeutic processes involving the stomatognathic } \\
\text { system. }\end{array}$} \\
\hline Accep & \\
\hline $\begin{array}{l}\text { Keywords: } \\
\text { apoptosis, } \\
\text { Bcl-2, Ki-67, p53, } \\
\text { follicular cyst, } \\
\text { keratocyst. }\end{array}$ & \\
\hline
\end{tabular}

\section{INTRODUCTION}

The term "apoptosis" is of Greek origin and originally means the falling of floral petals or tree leaves. It was used for the first time in 1972, by Kerr, Wylli and Currie to denote programmed cell death. As used, the term is applied to the removal of irreparable destroyed, infected or mutated cells from the organism, as well as the removal of excess normal cells. Both apoptosis and proliferation are processes that are critical for maintaining human homeostasis. An imbalance between these two processes can lead to the development of neoplastic processes. Apoptosis is an orderly and active process that is strictly controlled genetically. In most cases, it affects single cells.

Morphological and molecular changes in the process of apoptosis affect mostly the nucleus. Chromatin is condensed, and DNA is broken down by particular enzymes into fragments of 180-200 base-pairs. Apoptotic cells become smaller and the cytoskeleton is reorganised. The cellular membrane does not collapse, it remains intact and forms characteristic indentations. These lead to the breakdown of the cell into numerous apoptotic bodies containing fragments of the nucleus, cellular organella and cytoplasm. Phosphatidylserine then appears in the external layer of the cellular membrane. This enables the cell fragments to be recognised and phagocytised by macrophages without the presence of any inflammatory process.

There are several factors that influence the process of programmed cell death. These can be classified as endo- and

Corresponding author

e-mail: koszelj@wp.pl exogenic factors that activate or inhibit apoptosis. The process of apoptosis can be divided into three main phases: initiation, execution and disposal, and the execution and disposal phases have the same course in most cases. The initiation phase can, however, proceed according to two different pathways: the intrinsic pathway - mitochondrial, or extrinsic pathway - initiated by death signal transducers. The intrinsic route is initiated by direct interaction with the external mitochondrial membrane of, e.g. reactive oxygen species, damage to nuclear chromatin through uncontrolled proliferation of the cells as an effect of oncogene activation, and deactivation of suppressor genes, or corticosteroids. In the intrinsic path, the synthesis of the $\mathrm{Bcl}-2$ protein family begins (Bcl-2, Bax, Bad proteins) as a response to a damaging stimulus, which changes the permeability of the mitochondrial membrane. The Bcl-2 protein decreases membrane permeability, and Bax and Bad proteins increase membrane permeability. As the permeability of the external mitochondrial membrane increases, the cytochrome c moves to the cytosol, and it binds with the Apaf-1 protein, which leads to its activation, which, in turn, enables the binding of dATP. This induces the oligomerisation of 7 molecules of the Apaf-1 protein. A complex known as an 'apoptosome' is formed and it activates Caspase-9. The latter activates Caspase- 3 and Caspase-6 (effector caspases). The activation of the extrinsic pathway begins by binding specific ligands (protein death signals excreted by adjacent cells) to membrane FAS receptors (the so- called 'death receptors'). Death receptors are receptors of the TNF family. Binding the ligand by death receptors leads to its trimerisation. 
A receptor activated in this way binds (through a death domain) the adapter protein FADD or TRADD. The complex (the so-called 'DISC') activates Caspase-8. The latter activates Caspase-4, Caspase-9 and Caspase-11, all of which activate Caspase-3 and Caspase-9 (effector caspases). Both routes can influence each other by the BID protein, which, when cut by Caspase- 8 (the extrinsic pathway), opens the canals formed by the Bax protein blocked by the Bcl-2 protein (the intrinsic pathway), and enables the outflow of cytochrome $\mathrm{c}$ from the mitochondrion. The execution phase lasts approximately 30 minutes and begins when effector caspases become active. The most important caspase is caspase-3. It leads to the breakdown of the ICAD protein (an inhibitor of caspase-activated DNAse), which results in the activation of DNAse that cuts DNA into fragments; activation of the acinus protein, which leads to the condensation of the chromatin; and fragmentation of the nuclear lamin protein, which results in the fragmentation of the nucleus. In the disposal phase, apoptotic bodies undergo phagocytosis, and so the necrotic cell disappears $[1,3,4,10]$. The process of apoptosis have been subjected to numerous studies conducted on dental pulp cells, dental hard tissue cells, periapical tissues and alveolar bone.

Hertwig's epithelial root sheath (HERS) and epithelial cell rests of Malassez (ERM) undergo apoptotic processes during tooth development. As the roots develop, ectomesenchymal cells of the dental follicle migrate to Hertwig's sheath, they lose their epithelial structure and gradually differentiate into cementoblasts that produce cement. During that process, most epithelial cells of Hertwig's sheath remain in the periodontal ligament, but some of them undergo apoptosis just after the beginning of cement formation. Epithelial cells of Mallasez are groups of cells which are formed by epithelial Hertwig's root sheath that remain in the periodontal ligament for the organism's entire life. Nevertheless, with time, their number decreases due to the process of apoptosis. Malassez cells can be found near the surface of the root in the apical third of the periodontal ligament.

The process of apoptosis has also been observed in reduced ameloblasts in the area of the dental follicle during tooth eruption. Apoptosis of these cells is closely related to that in dental follicle cells and their derivatives: cementoblasts and cementocytes. Studies have shown that dental follicle cells and cementoblasts induced the apoptosis of ameloblasts and HERS and ERM cells. Probably, ameloblast apoptosis as induced by the cementoblasts is continuous and constant, and is not a temporary process. The outcomes suggest that active epithelial-mesenchymal interaction in the odontogenic epithelium, and nerve cells originating from the ectomesenchymal crest, induce the apoptosis of epithelial cells, whereas non-odontogenic epithelial cells do not interact with ectomesenchymal cells. Matalova believes that the apoptosis of ameloblasts, HERS and ERM induced by dental follicle cells and cementoblasts, occurs due to the activation of the Fas/Fas ligand/Caspase-8 [6,9].

Vermelin studied the presence of apoptotic cells in the dental pulp of intact human premolar teeth and rat molar teeth. The cells could be found in the peripheral zone of the pulp, mostly in the subodontoblastic layer, and there were considerably more of these in the coronal pulp than in the root canal pulp. The conducted studies suggest that most odontoblasts did not get labelled. A few nuclei with features characteristic for apoptosis were observed in rat molars in electron microscopy, i.e. nuclei with condensed chromatin and remains of cells being apoptotic bodies.

The results of electron microscopy examination of intact teeth suggest that apoptosis physiologically takes part in the regulation of the cell population of the pulp cells. Yet, the occurrence and the importance of apoptosis in the dental pulp are not fully known. Apoptotic cells were observed in peripheral layers of the pulp in subodontoblastic layers - more frequently in the coronal than in the radicular pulp. Cells which present a great ability to proliferate are much more susceptible to apoptosis than cells that have lost their ability to differentiate. Pulp cells undergo apoptosis, as they preserve the ability to multiply and differentiate in response to experimental exposition. In odontoblasts, however, which are post-mitotic cells, apoptosis has not been observed. As a result of constant dentine deposition, the volume occupied by pulp and odontoblasts decreases. This gradual reduction suggests that some odontoblasts must become necrotic as well. Vermelin's studies confirmed the presence of apoptosis in healthy pulp not affected by inflammatory processes, this suggests that it plays a role in the regulation of pulp cell population [22].

Apoptosis also occurs during elimination of pulp in physiological resorption of the roots of primary teeth. In the pulp of deciduous teeth being in the state of physiological resorption (at least 1/3 of root length), researchers have seen shrunken cells with condensed chromatin and condensed cytoplasm, frequent apoptotic bodies and no inflammatory reactions, which may suggest that apoptosis occurs in these pulp cells. In the pulp of permanent teeth, these processes were found to a much lesser extent. During physiological resorption, all root cells undergo atrophy (cementum, dentin, pulp). Cementum and dentin are also eliminated due to the action of odontoclasts and osteoclasts. The mechanism of pulp atrophy is not known. Studies have shown that apoptosis progresses more intensively in the pulp of primary teeth than in permanent teeth, hence, apoptosis probably takes part in pulp atrophy during root resorption [17].

The presence of apoptosis in periapical tissues and bone supporting the socket in rats treated with orthodontic appliances was evaluated. The highest number of apoptotic cells was observed on the third day after placement of the appliance. These were located mainly in the periapical area and in the pulp of the first and the second molar. The first molar was subject to direct pulling forces, and the second molar was subject to the forces indirectly because of the influence of the ligament between the first and the second molars [14].

An immunohistochemical analysis of apoptotic factors was conducted on the epithelium lining of radicular cysts. The epithelium of the cysts showed the expression of single-stranded DNA (ssDNA) in the basement membrane and in the superficial epithelial cells, while, Ki-67 was observed in the cells of the basal layer of the epithelium of the cysts. Both Ki-67 and ssDNA levels were slightly elevated in cysts with a thick epithelial lining under the influence of inflammatory processes. The Bax and Bcl-2 proteins were also found in a few cells of the basement 
layer of the epithelium, and Caspase- 3 was found in the superficial layer of the epithelial cells. The expression of $\mathrm{Ki}-67$ and ssDNA in Caspase-3-positive areas was considerably higher than in Caspase-3 negative areas. However, a significantly limited expression of Fas was observed in the epithelium. Conducted studies suggest that the markers of apoptosis take an active part in the physiopathological activity of inflammatory periapical tissues. Inflammation in these tissues, hence, modulates the reactivity of apoptotic factors $[18,19,20]$. Lloyola et al., found in their studies that apoptotic cells and apoptotic bodies could be found in the epithelium of all examined odontogenic cysts, and that the frequency of apoptosis was higher in the atrophic epithelium than in the hyperplastic epithelium $[8,20]$.

The role of apoptosis in pericoronal tissues of erupting third mandibular molars was studied by Edamatus et al. In order to explain the possible role of factors associated with apoptosis in pericoronal tissues, the expression of Fas, Bcl-2, $\mathrm{Ki}-67$ and ssDNA in the epithelial components of dental follicles (DF) and dentigerous cysts (DC) associated with third mandibular molars was examined. In their work, the expression of Fas and ssDNA was observed in the cell membrane and cytoplasm of superficial epithelial cells of DF and DC, and the expression of Bcl-2 and Ki-67 was seen in the cytoplasm of epithelial cells adjacent to the basement membrane. Of note, the expression of Bcl-2 was considerably lower in DF than in DC. Moreover, there were more ss-DNA-positive cells in DF, whereas more Ki-67-positive cells could be found in DC. Furthermore, the presence of inflammation led to a slightly higher level of ssDNA and Ki-67.

The presence of Fas and its ligand in dental follicles suggests that the Fas/Fas ligand system is related to tooth development. In the study, epithelial cells of DF and DC exhibited the expression of Fas in superficial layers, which might suggest that Fas can play a role in apoptotic death of these cells. The presence of Bcl-2 protein in epithelial cells adjacent to the basement membrane in DF and DC indicated that apoptotic reactions are suppressed by this protein in these areas. Considerably higher expression of the Bcl-2 protein in the cytoplasm of epithelial cells adjacent to the basement membrane in DC in comparison to DF suggests that this apoptosis inhibitor can act as a factor in the pathogenesis of DC development. Anti-ssDNA antibodies are a sensitive marker of apoptotic cells, especially of those in early stages of cell death. The study showed that ssDNA is expressed in the dispersed nuclei of superficial DF and DC cells, and by that, it shows areas of apoptotic death. $\mathrm{Ki}-67$ is a nuclear antigen present in all phases of the cellular cycle (i.e. G1, S, G2, M) and absent in the G0 phase. It is a credible marker of cellular proliferation. The marker was found in epithelial cells adjacent to the basement membrane in DF and DC. Although the differences in ss-DNA and $\mathrm{Ki}-67$ isolation in DF and DC were not significant, ssDNA was slightly higher in DF, whereas the expression of Ki-67 was higher in DC. The study has shown that the state of tooth impaction did not correlate with the expression of apoptosis-related factors. In DF, the expression of apoptosisrelated factors and proliferation markers is most probably modulated by the morphological features of epithelial components, as well as by inflammatory changes. This fact suggests that the states of tooth impaction do not have to reflect the physiopathological state of DF $[2,20]$.

The aim of the studies by Razavi et al. was the evaluation of the relationship between the size of the follicle of impacted third molars $(>=2.5 \mathrm{~mm}$ and $<2.5 \mathrm{~mm})$, and the rate of cell proliferation and apoptosis. Follicles whose size was $2.5 \mathrm{~mm}$ or more exhibited higher expression of Bcl-2 protein than follicles smaller than $2.5 \mathrm{~mm}$, which is the cause of apoptosis inhibition and increase in cell accumulation, and can lead to the development of cystic lesions, as well as odontogenic tumours. Furthermore, follicles with radiolucency higher than $2.5 \mathrm{~mm}$ exhibited increased immunoreactivity to $\mathrm{Bcl}-2$ protein. It is known that changes in proliferation rate and apoptosis of odontogenic epithelial cells can influence the development of odontogenic epithelial lesions, and the study confirms the thesis that the inhibition of apoptosis in the odontogenic epithelium of follicles of impacted third molars with radiolucency greater than 2.5 $\mathrm{mm}$ can lead to the development of cystic lesions or odontogenic neoplasms. The outcomes of the study suggest that impacted third molars with radiolucency of $2.5 \mathrm{~mm}$ or more can be connected with the deregulation of cell death $[13,16]$.

Similar conclusions were drawn by Rahman et al. who observed over-expression of $\mathrm{Bcl}-2$ and $\mathrm{Ki}-67$ proteins in the pericoronal tissues of impacted teeth with squamous metaplasia, and in DC. That study attempted to evaluate and compare proliferation rates in the epithelium surrounding the impacted third molars and dentigerous cysts (DC). Since the histopathological changes which appear during different stages of dental follicle development can lead to the development of DC and neoplasms, the expression of Bcl-2 and $\mathrm{Ki}-67$ proteins in some epithelial cells is believed to be a credible marker of cell proliferation and lesion aggressiveness. Excessive amounts of this marker can indicate a developing pathological lesion in the area of third molars before the lesion is detected radiographically. The work of Rahman et al. showed that the expression of Ki-67 in inflamed tissues was considerably higher than in tissues without inflammation. In conclusion, the outcomes of the study show that the absence of radiographic features is not enough to state the absence of a lesion. Moreover, the expression of anti-apoptotic and proliferative markers is modulated by the morphological features of epithelial components and by the presence of inflammatory changes $[12,13,20]$.

The study by Lin et al. explained in what way nitrogen (II) oxide modulates the apoptosis of macrophages and osteoblasts. These play a key role in the development of periapical lesions. The role of nitrogen (II) oxide (NO) in the process of apoptosis is still controversial, as it is believed that it can both stimulate and inhibit this process. Bone loss induced by bacteria, including periapical lesions, is one of the most common diseases of the bone. Lipopolysaccharide (LPS) mediates the transmission of numerous pathological effects of bacteria, and it is thought to be one of the most important osteolytic factors. In periapical lesions, there is a relationship between the LPS level and the rate of bone destruction. Nitric oxide released from macrophages stimulated by LPS increases the apoptosis of osteoblasts and macrophages. This contributes to the development of 
periapical lesions. These outcomes confirm the important role of macrophages in host defence mechanisms. They synthesise pro-inflammatory cytokines and reactive oxygen/ nitrogen species that are used to eliminate pathogens. Data suggest that the apoptosis of macrophages induced by LPS can contribute to the development of periapical lesions, and NO plays a key role in this process $[7,11]$.

Wu et al., in their study, tried to explain whether hydrogen peroxide could induce apoptosis in pulp cells. Previous work had shown that hydrogen peroxide can penetrate enamel and dentin, and it can reach the pulp chamber and the pulp. This process can occur during tooth bleaching or laser irradiation. In their work, in order to determine whether hydrogen peroxide induces apoptosis in dental pulp cells, the expression of cleaved caspase- 3 and caspase- 9 was assessed in vitro (previous work has shown that caspase- 8 initiates the receptor-ligand pathway and caspase- 9 initiates the mitochondrial pathway). Hydrogen peroxide is a small molecule that penetrates enamel, dentin and cell membrane, and it is known that it can induce apoptosis in periodontal ligament cells and pulp cells. However, it is believed that apoptosis prevents inflammatory lesions in these tissues. In this study, the expression of procaspase-3, cleaved caspase- 3 and active caspase- 9 was considerably higher in the group exposed to hydrogen peroxide. Caspase- 9 activation indicates that apoptosis in dental pulp cells induced by hydrogen peroxide occurs as a result of mitochondrial pathway activation. Apoptosis plays an important role in the formation of reparative dentine by creating space for new dentin, and it prevents the development of inflammation. This study confirms that hydrogen peroxide is cytotoxic for dental pulp cells and that it induces apoptosis through the caspase-9-dependent pathway, which provides the basis for pulp regeneration [21].

Rangiani and Motahhary assessed the expression of proand anti-apoptotic factors (Bax and Bcl-2 proteins) in Orthokeratinized Odontogenic Cysts (OOC) and Odontogenic Keratocysts (OKC). They concluded that the expression of bax protein in both cysts is comparable, and the expression of Bcl-2 protein was considerably higher in $\mathrm{OKC}$. The authors found that the relationship between Bcl-2 and bax correlated with the frequency of apoptosis, and that the higher it was, the higher the cell survival rate was and the more aggressive the lesions were. It was observed that the $\mathrm{Bcl}-2 / \mathrm{Bax}$ ratio is different in different layers of OKCs. In the basal cell layer of the cystic wall, a high expression of Bcl-2 protein results in a high cell survival rate, which leads to cyst recurrence in cases in which the cyst wall is not removed totally. However, in the higher layers of the cystic wall, the expression of Bax protein without the presence of Bcl-2 protein leads to a high percentage of cells undergoing apoptosis, which prevents the thickening of the cystic wall and the development of a tumour mass. Hence, Rangiani and Motahhary put forward that immunohistochemical evaluation of Bcl-2 protein expression in the walls of OOC and $\mathrm{OKC}$ can be used as a practical method of differentiation between these two cyst types $[15,20]$.

Similar results were obtained by Kichi et al. in the assessment of the anti-apoptotic factor Bcl-2 in the basal cell layer of OKC epithelium. The study also included an assessment of two factors influencing cell proliferation - the p53 and the
Ki-67 proteins. Their results revealed that over-expression of both parameters occurs in both the basal and suprabasal layers of the cyst lining [5,20].

In conclusion, we put forward that future studies should concentrate on how to use the knowledge of apoptotic processes and their inhibitors in therapeutic processes involving the stomatognathic system.

\section{REFERENCES}

1. Bielak- Żmijewska A.: Resistance to apoptosis mechanisms of neoplastic cells., Kosmos problemy nauk biologicznych., 52,2-3,2003

2. Edamatsu M., et all.: Apoptosis - related factors in the epithelial components of dental follicles and dentigerous cysts associated with impacted third molars of the mandible., Oral Surg Oral Med Oral Pathol Oral Radiol Endod., 99,17,2005

3. Filip A.: Apoptosis - programmed cell death., Cz I Almamater., $1,46,2003$

4. Filip A.: Apoptosis - programmed cell death., Cz II Almamater., $2,47,2003$

5. Kichi E., et all.: Cell proliferation, apoptosis and apoptosis-related factors in odontogenic keratocysts and in dentigerous cysts., J Oral Pathol Med., 34,280,2005

6. Lee J.H., et all.: Dental follicle cells and cementoblasts induce apoptosis of ameloblast- lineage and Hartwig's epithelial root sheath/ epithelial rests of Malassez cells through the Fas-Fas ligand pathway., Eur J Oral Sci., 29,120,2012

7. Lin S.K., et all.: Nitric oxide promotes the progression of periapical lesion via inducing macrophage and osteoblast apoptosis., Oral Microbiol Immunol., 24,22,2007

8. Loyola A. M., et all.: Apoptosis in epithelial cells of apical radicular cysts., International Endodondic Journal., 38, 465, 2005

9. Matalova E., et all.: Caspase-7 participates in differentiation of cells forming dental hard tissues., Develop. Growth Differ., 615,55,2013

10. Mitrus I., Missol- Kolka E., Szala S.: Pro-apoptotic genes in gene therapy of neoplasms., Współczesna Onkologia., 5,6,2001

11. Nagai H., et all.: Inducible nitric oxide synthase and apoptosis related factors in the synovial tissues of temporomandibular joints with internal derangement and osteoarthritis., American Association of Oral and Maxillofacial Surgeons J Oral Maxillofac Surg., 61, 801, 2003

12. Rahman F., et all.: Analysis of the immuno-expression of Ki-67 and bcl-2 in the pericoronal tissues of impacted teeth, dentinogerous cysts and gingiva using software image analysis., Dent Res. J., $1,10,2013$

13. Rakprasitkul S., et all.: Pathologic changes in the pericoronal tissues of un-erupted third molars., Oral Medicine.,

14. Rana M.W., et all.: Detection of apoptosis during orthodontic tooth movement in rats., American Journal of Orthodontics and Dentofacial orthopedics., 119,5,2001

15. Rangiani A., Motahhary P.: Evaluation of bax and bcl2 expression in odotogenic keratocysts and orthokeratinized odontogenic cysts: A comparison of two cysts., Oral Oncology., 2008

16. Razavi S.M., et all.: The relation of pericoronal third molar follicle dimension and bcl-2/ki-67 expression: An immunohistochemical study., Dent Res. J., 1,9,2012

17. Rodrigues L.V., et all.: Apoptosis in pulp elimination during physiological root resorption in human primary teeth., Braz. Dent. J., 20, 3, 2009

18. Suzuki T., et all.: Immunohistochemical analysis of apoptosis-related factors in lining epithelium of radicular cysts., J Oral Patho Med., 34, 46, 2005

19. Takahashi K., et all.: Cell synthesis, proliferation and apoptosis in human dental periapical lesions analysed by in situ hybridisation and immunohistochemistry., Oral Diseases., 320, 5, 1999

20. Toslos K. I., Kakarantza-Angelopoulou E., Kapranos N.: Immunohistochemical study of bcl-2 protein, Ki-67 antigen and p53 protein in epithelium of glandular odontogenic cysts and dentigerous cysts., J Oral Pathol Med., 29, 44, 2000

21. Wu T., et all.: Hydrogen Peroxide induces apoptosis in human dental pulp cells via Caspase-9 dependent pathway., JOE., 39,9,2013 
22. Vermelin L., Lecolle S., Septier D. et all: Apoptosis in human and rat dental pulp., Eur J Oral Sci., 547,104,1996 